\title{
Processo decisório em sistemas de saúde: uma revisão da literatura
}

\section{Decision making process in health systems: a literature review}

\section{Raquel Flexa}

Centro Federal de Educação Tecnológica Celso Suckow da Fonseca. Departamento de Engenharia de Produção. Rio de Janeiro, RJ, Brasil.

E-mail: raquelflexaळyahoo.com

\section{Correspondência}

Av. Maracanã, 229, bloco L, sala L29. Rio de Janeiro, RJ, Brasil. CEP 2027ı-IIO.

\section{Resumo}

Sistemas de saúde podem ser compreendidos como sistemas sociais que apresentam uma combinação de recursos, organização, financiamento e gerência que visam oferecer serviços de saúde para uma população. Para Tanios et al., a tomada de decisão em sistemas de saúde envolve a definição de critérios e valores, além do uso de evidências e do julgamento de alternativas na formulação de políticas públicas em saúde. O objeto deste artigo é a realização de uma revisão bibliográfica sobre o processo decisório em sistemas de saúde, como forma de auxiliar e orientar futuras pesquisas sobre o tema. Para isso, foi realizada a busca bibliográfica nas bases ISI Web of Knowledge e Scopus, sem restrição quanto ao período de publicação. A partir disso, foi feita a redação sintóptica dos conteúdos extraídos das publicações levantadas nessa busca. Os resultados dessa revisão foram agrupados em quatro temas: definições e contexto do processo decisório em sistemas de saúde; critérios e desafios do processo; modelos de tomada de decisão; e informações e sistemas de informação de suporte ao processo decisório.

Palavras-chave: Sistemas de Saúde; Saúde Pública; Tomada de Decisão; Processo Decisório. 


\section{Introdução}

Health systems are social systems that combine different types of resources, organization structures, funding models and management mechanisms to provide health services to a defined population. Tanios et al. say that the decision-making process in a health system deals with the definition of criteria and values, use of evidences and evaluation of health policies. In this context, this research aims to conduct a literature review about the decisionmaking process in health systems, to assist and guide future studies. In this regard, a bibliographic search was made in two different databases: ISI Web of Knowledge and SCOPUS. Publications found in this search were summarized through a superficial reading and the results were grouped into four themes: definitions and context in the decision-making process in health systems; criteria and challenges in this process; decision-making models; and information and information systems to support decision makers.

Keyword: Health System; Public Health; Decision Making; Decision Making Process.
De acordo com o entendimento de Bispo Júnior e Messias (2005), os sistemas de saúde podem ser compreendidos como sistemas sociais que apresentam uma combinação de recursos, organização, financiamento e gerência que visam oferecer serviços de saúde para uma população. Segundo os autores, independentemente da forma de organização social e econômica, a maioria dos sistemas têm objetivos comuns, como o alcance de um nível ótimo de saúde, distribuído de forma equitativa; a garantia de uma proteção adequada dos riscos para todos os cidadãos; o acolhimento dos cidadãos; e a efetividade e eficiência dos serviços de saúde.

Santos (2009), adicionalmente, considera que um sistema de saúde é definido a partir de suas leis e regulações, forma de financiamento, lógicas de administração e do escopo de sua produção dos serviços e bens de saúde para o atendimento a determinada população. Os sistemas de saúde apresentam uma vasta combinação de mecanismos decisórios e articulações políticas que resultaram em formatos institucionais bastante heterogêneos quanto às formas de controle e regulação, à definição de atribuições e às formas de financiamento (Biasoto Júnior; Silva; Dain, 20o6).

Nesse sentido, Wickremasinghe et al. (2016) definem o processo de tomada de decisão em sistemas de saúde enquanto um conjunto de passos estruturados que inclui a busca pelo consenso entre as partes envolvidas e incorpora o uso de informações coletadas nos níveis locais para tratar situações complexas, levando em consideração as especificidades do contexto e chegando ao desenvolvimento e à avaliação de soluções inovadoras. Para Tanios et al. (2013), a tomada de decisão envolve a definição de critérios e valores, além do uso de evidências e do julgamento de alternativas na formulação de políticas públicas em saúde.

A complexidade do processo decisório em sistemas de saúde é ampliada dado o envolvimento de um grande número de atores, de diversas naturezas (técnicos, gestores, políticos, agentes sociais), em diferentes níveis de atuação (local, municipal, 
estadual e federal), com diferentes objetivos ou entendimentos sobre qual a melhor solução para um problema estabelecido.

Dados os pontos expostos, o objetivo deste artigo é a realização de uma revisão bibliográfica sobre o processo decisório em sistemas de saúde, como forma de auxiliar e orientar futuras pesquisas sobre o tema. Para isso, esta publicação apresenta o método utilizado na busca bibliográfica e, em seguida, expõe os principais conteúdos extraídos através da leitura sintóptica dos artigos, dissertações, teses e demais documentos levantados.

\section{Método}

Para Gil (2008), a pesquisa bibliográfica pode ser entendida como um processo que envolve as seguintes etapas: escolha do tema; levantamento bibliográfico preliminar; formulação do problema; elaboração do plano provisório de assunto; busca das fontes; leitura do material; fichamento; organização lógica do assunto; e redação do texto.

Tendo como critério para escolha das fontes de pesquisa a busca por bases de periódicos de maior impacto bibliométrico no mundo, optou-se por utilizar nesta pesquisa as bases ISI Web of Knowledge e Scopus.

Em ambas as bases as palavras-chave utilizadas foram "decision making process" e "health system", de forma conjugada (operador booleano "and"), sem restrição quanto ao ano ou tipo de publicação. Na base Scopus, retornaram 275 trabalhos que tratavam sobre o tema. Após a leitura dos resumos, verificou-se que apenas 36 trabalhos tinham relação direta com o objeto desta pesquisa. Nessa base, observou-se a existência de muitos trabalhos relacionados com o processo de tomada de decisão para o tratamento de doenças específicas, focando no processo decisório com base em evidência. Essa temática se afasta do objetivo desta pesquisa, dado que se busca o entendimento do processo decisório gerencial, com foco na organização e na gestão dos sistemas de saúde.

O mesmo mecanismo de busca foi aplicado na base ISI Web of Knowledge, e retornaram 86 publicações. Ao realizar a leitura dos resumos, utilizando o mesmo critério de filtro aplicado à leitura das publicações da base Scopus, verificouse a existência de 17 publicações aderentes a esta pesquisa. No entanto havia uma sobreposição de 6 artigos que apareciam em ambas as bases, reduzindo o número de novos artigos para 11. O resumo dos resultados da busca bibliográfica pode ser visto na Tabela 1.

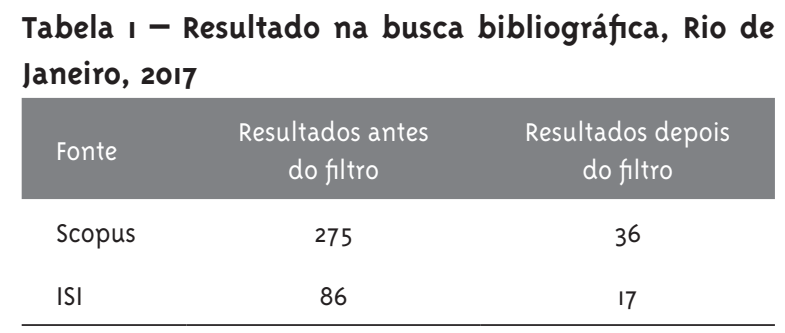

Os conteúdos extraídos a partir da leitura sintóptica das publicações encontradas na busca bibliográfica encontram-se sintetizados no item a seguir.

\section{Resultados}

Os resultados encontram-se estruturados em quatro grupos de assuntos que foram identificados na busca bibliográfica. 0 primeiro grupo trata das definições sobre o processo decisório em sistemas de saúde e o contexto no qual esse processo ocorre. O segundo grupo explicita os principais critérios utilizados no processo decisório, além de expor alguns desafios nesse curso. 0 terceiro bloco traz os modelos orientadores para a tomada de decisão e, por fim, o último grupo mostra a importância da informação e dos sistemas de informação nesse processo.

\section{Definições e contexto para a tomada de decisão}

Wickremasinghe et al. (2016) definem o processo de tomada de decisão em sistemas de saúde enquanto um conjunto de passos estruturados que inclui a busca pelo consenso entre as partes envolvidas e incorpora o uso de informações coletadas nos níveis locais para tratar situações complexas, levando em consideração as especificidades do contexto e chegando ao desenvolvimento e à avaliação de soluções inovadoras. 
Para Tanios et al. (2013), a tomada de decisão envolve a definição de critérios e valores, além do uso de evidências e do julgamento de alternativas na formulação de políticas públicas em saúde. Adicionalmente, Schroeder (2016) percebe que as pesquisas atuais enfocam a discussão sobre a avaliação do impacto das políticas públicas ou apenas a identificação das necessidades para a formulação de políticas. A autora defende, no entanto, que é necessário aprofundar o entendimento quanto ao processo de tomada de decisão em si.

Wickremasinghe et al. (2016) perceberam que há uma forte concentração de estudos sobre tomada de decisão em saúde pública que têm como foco a definição quanto às prioridades para alocação de recursos ou métodos para elaboração de planejamento e orçamento para as ações. Um ponto de destaque dos autores é a verificação de que mais de $80 \%$ dos estudos por eles avaliados descrevem o processo de tomada decisão como um mecanismo de construção de consenso entre os atores envolvidos, independente dos passos propostos no processo de tomada de decisão. Outro destaque desse estudo foi a carência de modelos que trouxessem o debate sobre a avaliação da efetividade das decisões tomadas e das ações implementadas nesse processo.

Segundo Mackenbach e McKee (2015), há um crescente número de estudos sobre o impacto das decisões políticas no processo decisório em sistemas de saúde. Para aprofundar o conhecimento sobre o processo de tomada de decisão nesse contexto, os autores definiram quatro grupos de condições políticas que afetam esse processo. 0 primeiro grupo se refere à participação popular na tomada de decisão, através da qual as preocupações populares podem ser expressadas e levadas em consideração pelos tomadores de decisão. Esse grupo pode ser entendido como "qualidade da estrutura e do processo democrático” (Mackenbach; McKee, 2015).

O segundo grupo trata da representação política nesse processo, que pode ser entendida por aspectos como o comparecimento dos eleitores às urnas e a orientação política do eleitorado. O terceiro grupo responde pela distribuição de poder nas democracias, que pode ser compreendida através de fatores como o número de partidos políticos, a relação de poder entre os poderes Executivo e Legislativo e os mecanismos de representação de grupos de interesse no processo democrático (Mackenbach; McKee, 2015).

O quarto grupo diz respeito à efetividade do governo. Os autores defendem que, embora o perfil político dos tomadores de decisão influencie diretamente no desenho das políticas públicas, é a "qualidade da máquina governamental" que determina se essas políticas serão plenamente implantadas (Mackenbach; McKee, 2015). Esse grupo é diretamente influenciado por fatores como profissionalização do serviço público, estabilidade política e institucional, e níveis de corrupção.

Como resultado de seu estudo, Mackenbach e McKee (2015) observaram que há uma forte correlação positiva do primeiro grupo (qualidade da estrutura e do processo democrático) e do quarto grupo (efetividade do governo) com indicadores de saúde pública. O segundo grupo (representação política) e o terceiro (distribuição de poder), por sua vez, não mostraram correlações fortes com esses indicadores de mensuração da implementação das políticas de saúde.

Outra característica da configuração política que influencia o processo de tomada de decisão em sistemas de saúde é o federalismo e a descentralização do poder. Wickremasinghe et al. (2016) destacam que, em sistemas de saúde com gestão descentralizada, característica comum em sistemas federalistas de governo, há a necessidade de discussão quanto ao grau de liberdade dos gestores locais de saúde no processo decisório. Os autores defendem que uma baixa alocação de direitos de decisão para os gestores locais impede que sejam tomadas decisões que realmente atendam às necessidades locais da população.

Programas e ações governamentais, em geral, são testados em pequena escala e depois têm seu escopo ampliado. Nesse sentido, Milat et al. (2014) estudam o processo de tomada de decisão para o "aumento de escala" (scale up) de programas de saúde e perceberam que essa decisão se dá através da interação entre formuladores de políticas públicas (policy makers), profissionais da área de saúde e, por último, com a aprovação das lideranças políticas e instituições que atuam na subvenção e/ou no financiamento de programas de saúde. Os formuladores de políticas públicas descrevem 
esse processo como uma construção de planos de ação, com o apoio de lideranças políticas nos poderes Executivo e Legislativo, além de contribuições de conteúdo de diversos atores, como comitês, grupos de trabalho, opinião de especialistas, pesquisadores e acadêmicos.

As decisões se dão em um contexto fortemente influenciado por fatores políticos que, por isso, podem rapidamente mudar ou ser alteradas em função de valores individuais, valores partidários ou experiências do grupo tomador de decisão (Milat, King et al., 2014). Para os formuladores de políticas públicas em saúde, os principais aspectos que influenciam a tomada de decisão são o alinhamento com políticas governamentais, a disponibilidade de recursos financeiros, o suporte das lideranças políticas e o suporte de outros atores (stakeholders) diretamente envolvidos na saúde pública. Além disso, os autores destacam a ausência de estudos sobre custos de implementação e sobre a relação entre custo e efetividade para que seja tomada a decisão quanto ao aumento de escala de programas de saúde.

Um ator que contribui ativamente no processo de tomada de decisão em sistemas de saúde é o meio acadêmico, através de suas pesquisas. Choi et al. (2016) buscam entender como esse ator pode influenciar na tomada de decisão e focam sua pesquisa em dois países: Canadá e China. Os autores observaram que os formuladores de políticas em ambos países concordam que as melhores formas para que haja uma efetiva interação entre esses dois mundos são (1) o desenho das pesquisas acadêmicas com base nas necessidades de políticas públicas e (2) a existências de fóruns que permitam o debate entre pesquisadores acadêmicos e formuladores de políticas públicas em saúde.

Quanto ao primeiro ponto - desenho das pesquisas baseado nas necessidades políticas -, os autores (Choi et al., 2016) ressaltam que o padrão seguido até então é de concepção e realização de pesquisas, sem a necessária interação com os formuladores de políticas públicas. Posteriormente, os resultados da pesquisa são disseminados através de artigos e publicações científicas. Choi et al. (2016), entretanto, apontam que as necessidades das políticas públicas devem orientar a concepção das questões das pesquisas científicas, levando assim o pesquisador para o tratamento de problemas do "mundo real". Ou seja, a interação entre academia e formulação política deve mudar da formulação de estratégias de disseminação de conhecimento (back end ou downstream) para a concepção do conhecimento (front end ou upstream), através da efetiva implementação de instrumentos de cooperação entre esses dois universos.

\section{Critérios e desafios no processo de tomada de decisão em sistemas de saúde}

Tanios et al. (2013) afirmam que, com o aumento dos gastos com saúde e a exposição dos pacientes a novas tecnologias, cresce a necessidade de se explicitar os critérios usados no processo de tomada de decisão para a definição de prioridades de investimentos e os critérios para alocação de recursos. Para Phelps et al. (2016), os recursos escassos disponíveis para o atendimento aos sistemas de saúde realçam a importância de um processo de tomada de decisão estruturado a partir de um planejamento estratégico, com o uso de critérios que devem ir além das análises de custo e efetividade.

Corroborando o estudo de Phelps et al. (2016), Tanios et al. (2013) reforçam que o paradigma do julgamento com base nas análises de custo e efetividade das soluções em saúde é limitado e, por isso, a elucidação dos demais critérios nesse processo é a chave para garantir sustentabilidade, equidade e eficiência dos sistemas de saúde. A falta de transparência, por sua vez, pode criar potenciais conflitos entre as partes envolvidas na tomada de decisão.

Adicionalmente, Phelps et al. (2016) defendem que a análise multicritério aplicada ao processo decisório na saúde pública aumenta a flexibilidade, a transparência e gera melhores subsídios para os envolvidos nesse processo. Além disso, os autores acreditam que a importância de ferramentas mais sofisticadas de apoio à tomada de decisão será aumentada à medida que os gastos com a saúde aumentarem e os recursos se tornarem mais escassos.

Para aprofundar o entendimento sobre os critérios para a tomada de decisão em saúde pública, 
Tanios et al. (2013) realizaram um estudo que envolveu 23 países e entrevistados que ocupavam cargos tanto na formulação de políticas públicas em saúde quanto na gestão e no atendimento na rede de saúde. Os 10 critérios apontados como os mais utilizados no processo de tomada de decisão foram: eficácia e efetividade clínica; segurança do paciente; qualidade da evidência; risco da doença para a vida do paciente; severidade da doença; indicações da intervenção nos protocolos clínicos; priorização de medidas curativas; impacto da intervenção nos custos; exigências organizacionais; e competências individuais.

Entretanto, houve uma diferença significativa entre os respondentes em cinco critérios caracterizados como "atualmente utilizados" versus aqueles apontados na categoria dos que "deveriam ser utilizados" no processo de tomada de decisão. Ou seja, são critérios que deveriam ser considerados no processo de tomada de decisão, mas que, efetivamente, não são levados em conta. Esses critérios são: impacto ambiental da intervenção; impacto da intervenção em outros serviços de saúde; custos da intervenção para o paciente; impacto da intervenção na produtividade; impacto da intervenção no empobrecimento do paciente (Tanios et al., 2013).

Além disso, o estudo focou em três grupos diferentes de respondentes: gestores, profissionais de saúde e pesquisadores. Observando os resultados da pesquisa de Tanios et al. (2013), é possível perceber que há discordâncias entre os grupos quanto aos critérios que devem ser considerados e àqueles que são de fato considerados na tomada de decisão. As diferenças entre as percepções podem ser vistas na Figura 1.

\section{Figura I - Critérios com diferenças significativas entre grupos de respondentes}

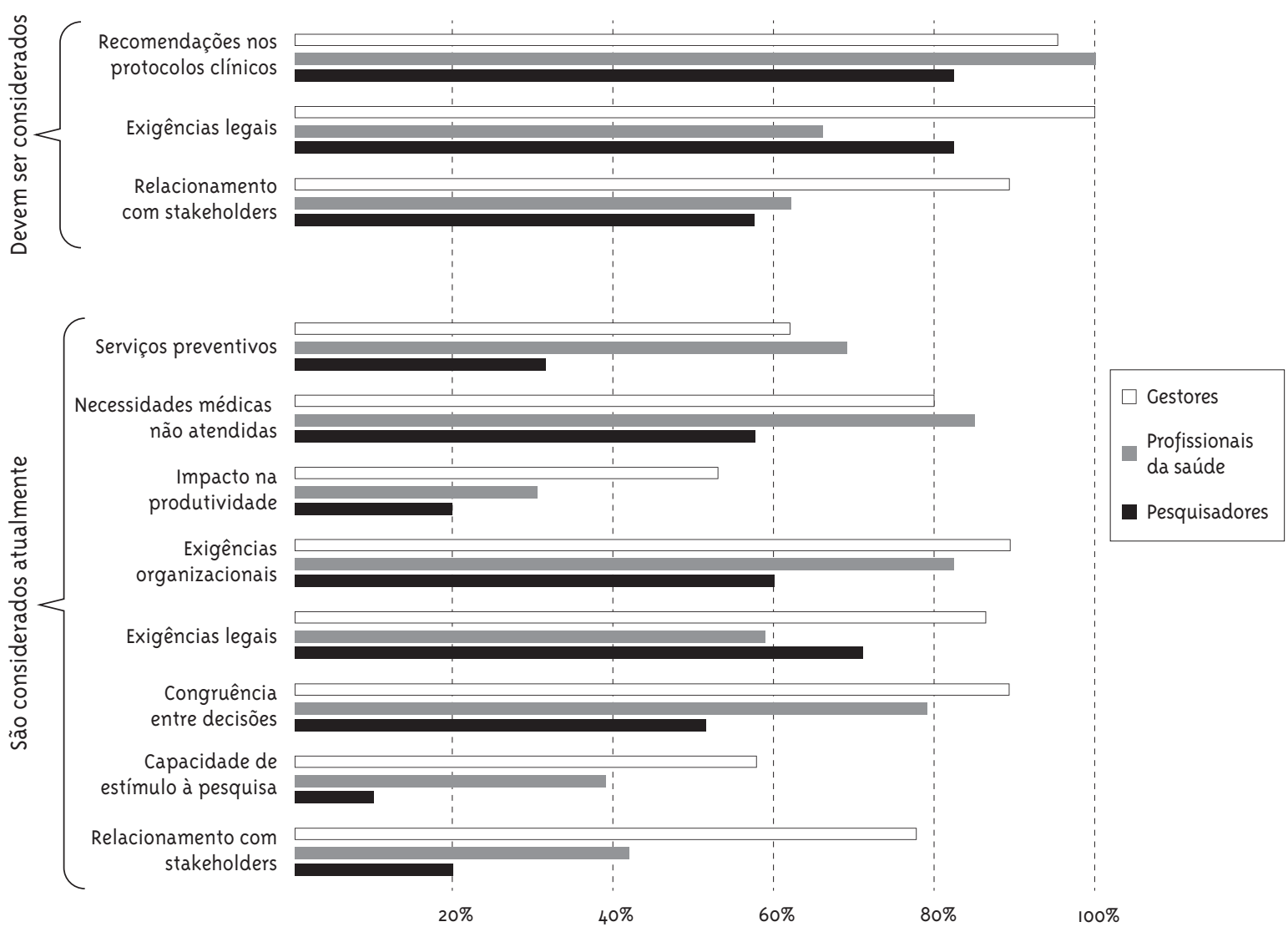

Fonte: Tanios et al., 2013 
O único critério no qual a proporção de respostas "deveria ser considerado" foi menor do que "é atualmente considerado" foi o de pressão e interesse de stakeholders. Para os autores, isso mostra o desconforto dos respondentes quanto ao papel que esse critério tem no processo decisório, uma vez que reduz a transparência e pode criar viés na decisão (Tanios et al., 2013).

Mesmo com a explicitação dos critérios para a tomada de decisão, Wickremasinghe et al. (2016) destacam que há ainda alguns desafios no processo de tomada de decisão em saúde pública, como:

- Disponibilidade, qualidade e uso das informações do sistema de saúde: há falta de informações nos níveis subnacionais e, quando elas existem, há dificuldade para acessá-las. Em alguns casos, as informações não são confiáveis ou não são disponibilizadas no momento adequado para a tomada de decisão;

- Dinâmicas sociais e políticas no processo de tomada de decisão: há a preocupação de que as decisões não sejam sempre baseadas nas informações, mas fortemente guiadas pelas pressões de grupos sociais e políticos. Além disso, os mecanismos de participação popular no processo de decisão ainda são frágeis e pouco utilizados. Há ainda os casos em que as decisões são baseadas nas prioridades nacionais, ao invés de revelar as reais necessidades locais;

- Decisões comprometidas por restrições orçamentárias: embora seja evidente que o processo de tomada de decisão tenha que ser cercado por restrições orçamentárias, os autores destacam aqui a inflexibilidade para remanejamento orçamentário durante a execução das ações quando há diferenças entre o planejamento original e a execução, além de situações em que há atrasos nos repasses de recursos financeiros para os entes subnacionais, mesmo já estando aprovados previamente em orçamento, comprometendo assim a execução das ações.

\section{Modelos de processo decisório em sistemas de saúde}

Wickremasinghe et al. (2016) realizaram uma vasta revisão bibliográfica sobre modelos de tomada de decisão em sistemas de saúde e conseguiram identificar 14 estudos que explicitam os passos estruturados ou semiestruturados para conduzir esse processo. Além dos modelos expostos por Wickremasinghe et al. (2016), ainda foi possível encontrar duas outras pesquisas que trazem contribuições para o entendimento sobre modelos de processo de tomada de decisão em saúde pública. Esse tópico tem como objetivo apresentar resumidamente as características dos modelos que se mostraram mais estruturados quanto à definição de etapas e responsabilidades, além de serem mais recentes (a partir do ano 200o), em um total de cinco modelos.

\section{Modelo de Heinonen et al. (2000)}

0 modelo de Heinonen et al. (200o) tem como preocupação a conexão entre o governo central e governos locais na implementação de políticas públicas em saúde, além de considerar a participação de outros agentes nesse processo, como organizações não governamentais (ONGs) e outras organizações situadas na comunidade local.

O modelo tem como partida a abordagem das Necessidades Básicas Mínimas (Minimum Basic Needs - MBN), que é um conceito desenvolvido nos anos 1970 por várias agências internacionais, visando não somente ao atendimento às necessidades básicas da população, como também o estabelecimento de bases para a participação popular no desenvolvimento de políticas públicas. O modelo de Heinonen et al. (200o) propõe o uso do MBN para garantir a autonomia dos governos locais, além de aumentar a colaboração e coordenação entre ONGs, comunidade, organizações locais e governo local, bem como encorajar a participação popular no planejamento e na implementação de projetos.

O primeiro passo do modelo é a formação de um grupo de trabalho, convocado pelo governo local, com planejadores municipais e representantes das secretarias de saúde, agricultura e desenvolvimento social. Ocorre então a capacitação de voluntários, 
líderes comunitários e grupos de interesse na abordagem MNB. O terceiro passo é a identificação das necessidades básicas não atendidas a partir de levantamento de evidências. Essas evidências serão então tratadas e analisadas pelos voluntários, com suporte do grupo de trabalho. 0 resultado dessa análise deve ser compilado e apresentado em fórum público. Para fins de priorização, deve ser realizado um ranqueamento das necessidades não atendidas, de acordo com os critérios selecionados pela comunidade. A partir o resultado da priorização e da seleção, são elaborados os planos de ação e haverá sua implementação.

\section{Modelo de Mubyazi et al. (2004)}

O modelo de Mubyazi et al. (2004) se foca na definição de prioridades para o planejamento anual das atenções primária e secundária pelos governos locais. O método apresenta cinco passos, e o primeiro deles é a identificação dos problemas de saúde pelas equipes dos conselhos locais de saúde, com o uso de manuais e diretrizes produzidos pelo governo central. Com base nessas informações, há o desenvolvimento do plano de saúde local, envolvendo as secretarias de saúde, de planejamento e de fazenda. Esse plano deve ser validado pelo conselho estadual ou distrital de saúde, e as revisões necessárias apontadas por esse grupo devem ser feitas pelas equipes locais. Por fim, o plano é submetido ao Ministério da Saúde para aprovação e reserva de recursos orçamentários e financeiros.

\section{Modelo de Maluka et al. (2010, 2011a, 2011b)}

O modelo de Maluka et al. (2010, 2011a, 2011b) trata da definição de prioridades para alocação de recursos na saúde pública e defende que esse processo deve ser embasado em evidências, premissas e princípios aceitos pelas partes envolvidas (stakeholders) e relevantes para o atendimento às necessidades da população. Para a definição de prioridades, os autores propõem o uso do modelo A4R: relevância (relevance), publicidade (publicity), recursos (appeals) e cumprimento (enforcement).

O primeiro passo do modelo é a definição de prioridades em sete áreas da saúde pública: saúde infantil e reprodutiva; doenças transmissíveis; doenças não transmissíveis; tratamento de doenças endêmicas; promoção da saúde; reforço de capacidades; e estrutura organizacional na gestão dos serviços de saúde. Essa priorização deve ocorre com base em dados epidemiológicos, estatísticas do sistema de saúde, pesquisas sobre necessidades das unidades de saúde e comunidade. Para as ações prioritárias, devem ser avaliadas a importância, a severidade, a viabilidade e os custos envolvidos. Essas prioridades e os argumentos para sua escolha devem ser divulgados para a comunidade local, e então os planos de ação e orçamento podem ser desenvolvidos. Esses planos e orçamento devem ser revistos pelos conselhos de saúde para garantir que contemplem efetivamente as necessidades da população. As ações serão então implementadas e continuamente monitoradas e avaliadas, com participação popular ao longo desse processo (Maluka et al., 2010, 2011a, 2011b).

\section{Modelo de La Vincente et al. (2013)}

O modelo de La Vincente et al. (2013) enfoca a definição de prioridades de investimentos e alocação de recursos, via definição de orçamento, pelos governos locais, especialmente no caso da saúde materno-infantil. Trata-se de um método estruturado de resolução de problemas que busca identificar, priorizar, selecionar e desenvolver estratégias para a resolução de questões prioritárias em saúde pública através da estimativa de custos e de impacto.

O primeiro passo do modelo é o levantamento de evidências críticas relacionadas à performance do sistema de saúde. Em seguida, o modelo propõe a análise sistemática e estruturada das causas raiz dos problemas relacionados à saúde maternoinfantil para as populações carentes. Após esse passo, há a identificação das estratégias viáveis para o tratamento das causas raiz e a estimativa do aumento da cobertura dos serviços de saúde com essas soluções. A etapa seguinte trata da estimativa do impacto e dos custos associados à implementação das alternativas de solução através de modelos epidemiológicos e econômicos para dar suporte à tomada de decisão. 0 resultado dessa fase irá então orientar a definição de prioridades e, posteriormente, a elaboração do planejamento e do orçamento (La Vincente et al., 2013). 
Modelo de Phelps et al. (2016)

Phelps et al. (2016) aproximam o processo decisório em saúde pública de um ciclo de planejamento estratégico. Os autores defendem, entretanto, que o processo difere do método geral aplicado a empresas privadas uma vez que deve considerar as necessidades específicas de uma população, o ônus causado pelo tratamento das doenças e as intervenções associadas. Assim como Gautham et al. (2016), Phelps et al. (2016) destacam a importância da disponibilidade de informações confiáveis e acuradas para suportar o planejamento em saúde pública.

Para os autores, o processo de tomada de decisão deve responder aos seguintes pontos:

- definição dos indicadores que devem ser monitorados, ou seja, quais são os objetivos do plano;

- critérios de alocação de esforços e avaliação de como essa escolha interfere no atendimento de um objetivo em detrimento de outros;

- consideração quanto aos trade-offs entre os possíveis esforços, ou seja, como decidir em situações em que a adoção de uma medida implica em um impacto deletério em outros aspectos da saúde pública.

Além disso, Phelps et al. (2016) defendem o uso de ferramentas multicritério, através de atribuição de pesos conforme a importância de cada atributo, para suportar o processo de tomada de decisão, uma vez que elas permitem o tratamento de diversas variáveis que trazem complexidade para o tomador de decisão. Uma vantagem na adoção de ferramentas dessa natureza é o aumento da transparência e da clareza que elas trazem ao processo, facilitando assim o consenso entre as partes envolvidas na tomada de decisão.

\section{Sistemas de informação no processo de tomada de decisão}

Para Gautham et al. (2016), sistemas de informação na gestão de sistemas de saúde são importantes ferramentas para seu planejamento e para o monitoramento de indicadores de desempenho em saúde. Esses sistemas requerem informações relacionadas a infraestrutura e recursos humanos, desempenho na entrega dos serviços, informação quanto à alocação e ao uso dos recursos financeiros, assim como dados efetivos das condições de saúde da população atendida.

Wickremasinghe et al. (2016) afirmam que sistemas de informação nos sistemas de saúde produzem uma grande quantidade de informações sobre a provisão dos serviços e sobre a saúde da população, permitindo assim que o processo de tomada de decisão descentralizado nos níveis estaduais, distritais e municipais seja melhor embasado e atenda melhor às necessidades da população local.

Para Gautham et al. (2016), as barreiras para o baixo uso dos sistemas de informação na saúde pública são problemas na qualidade das informações (dados incompletos, duplicados e de baixa confiabilidade), tecnologia para a coleta e consolidação dos dados, além das falhas relacionadas à tecnologia de comunicação dos dados.

Para Nutley, Macnabb e Saletine (2013), a qualidade das informações é fundamental para embasar o processo de tomada de decisão na busca pela melhoria dos sistemas de saúde. No entanto os autores ressaltam que a formulação e a reestruturação de programas de saúde ainda são realizadas sem o uso de dados adequados. Para eles, uma das principais razões para o processo decisório guardar essas características é que os sistemas de informação na saúde pública são fragmentados, complexos e não atendem plenamente às necessidades dos tomadores de decisão.

Wickremasinghe et al. (2016) acreditam que a qualidade da informação é afetada porque os agentes que geram as informações e aqueles que efetivamente são os usuários destas são grupos diferentes. Por conta dessa distância entre os grupos, há entendimentos diferentes quanto à importância dos dados coletados e sobre o impacto do uso de dados de baixa acurácia no processo decisório.

\section{Considerações finais}

Esse artigo teve como objetivo expor os resultados da revisão bibliográfica sobre processo decisório em sistemas de saúde. Após a realização 
da busca nas bases ISI Web of Knowledge e Scopus, esses resultados foram agrupados em quatro grupos de assuntos: (1) definições e contexto do processo decisório em sistemas de saúde; (2) critérios e desafios do processo; (3) modelos de tomada de decisão; e (4) informações e sistemas de informação de suporte ao processo decisório.

Observando os critérios de tomada de decisão, bem como os passos propostos nos modelos apresentados, é possível perceber que muitos estudos têm como foco a definição quanto às prioridades para alocação de recursos ou os métodos para elaboração de planejamento e orçamento para as ações (Wickremasinghe et al., 2016). Embora o processo decisório seja marcado pela utilização de critérios ainda limitados, a elucidação destes é a chave para garantir a sustentabilidade, a equidade e a eficiência dos sistemas de saúde, além de reduzir potenciais conflitos entre as partes envolvidas na tomada de decisão (Tanios et al., 2013).

Nesse contexto, percebe-se a necessidade de ferramentas mais sofisticadas de apoio à tomada de decisão. Para isso, Phelps et al. (2016) defendem que a análise multicritério aplicada ao processo decisório aumenta a flexibilidade, a transparência e gera melhores subsídios para os envolvidos nesse processo.

Quanto aos atores envolvidos no processo, verifica-se que a decisão se dá em um contexto fortemente influenciado por fatores políticos e, por isso, pode ser alterada em função de valores individuais, valores partidários ou da experiência do grupo tomador de decisão (Milat et al., 2014), mesmo com a coexistência de atores de diversas naturezas, esferas de atuação e interesses/objetivos sobre o assunto.

Adicionalmente, Wickremasinghe et al. (2016) sintetizam três principais desafios envolvidos no processo decisório em sistemas de saúde: (1) disponibilidade, qualidade e uso das informações do sistema de saúde; (2) dinâmicas sociais e políticas no processo de tomada de decisão; e (3) decisões comprometidas por restrições orçamentárias. Nutley, Macnabb e Saletine (2013) concordam que a disponibilidade das informações é uma fragilidade no processo decisório e, segundo eles, uma das suas principais razões está na fragmentação dos sistemas de informação, na sua complexidade e no fato de não atenderem plenamente às necessidades dos tomadores de decisão.

Embora o processo decisório em sistemas de saúde seja marcado pela necessidade de consenso (Wickremasinghe et al., 2016), a pesquisa permitiu perceber a ausência de publicações sobre as estruturas e os mecanismos de governança ao longo desse processo. Mostra-se necessário entender os papéis, as reponsabilidades, os direitos de decisão, os instrumentos e os mecanismos de apoio à tomada de decisão, bem como os fóruns e as estruturas organizacionais nos quais o processo acontece. Essa situação se agrava em um sistema federalista como o brasileiro, no qual os membros ocupam juridicamente o mesmo plano hierárquico e possuem autonomia relativa (Batista et al., 2011).

\section{Referências}

BATISTA, S. et al. O papel dos dirigentes municipais e regionais na criação e gestão dos consórcios públicos. Brasília, DF: [s.n.], 2011.

BIASOTO JÚNIOR, G.; SILVA, P. L. B.; DAIN, S. Regulação do setor saúde nas Américas: as relações entre o público e o privado numa abordagem sistêmica. Brasília: Organização Pan-Americana da Saúde, 2006. (Série técnica desenvolvimento de sistemas e serviços de saúde; 13)

BISPO JÚNIOR, J.; MESSIAS, K. Sistemas de serviços de saúde: principais tipologias e suas relações com o sistema de saúde brasileiro.

Revista Saúde.com, Jequié, v. 1, n. 1, p. 79-89, 2005.

CHOI, B. C. C. et al. Bridging the gap between science and policy: an international survey of scientists and policy makers in China and Canada. Implementation Science, London, v. 11, p. 1-11, 2016. Artigo 16.

GAUTHAM, M. et al. District decision-making for health in low-income settings: a qualitative study in Uttar Pradesh, India, on engaging the private health sector in sharing health-related data.

Health Policy and Planning, Oxford, v. 31, p. ii35ii46, 2016. Suplemento 2.

GIL, A. Como elaborar projetos de pesquisa. 4. ed. São Paulo: Atlas, 2008. 
HEINONEN, T. et al. Conceptualizing and applying a minimum basic needs approach in southern Philippines. Health Policy and Planning, Oxford, v. 15, v. 2, p. 194-206, 2000.

LA VINCENTE, S. et al. Supporting local planning and budgeting for maternal, neonatal and child health in the Philippines. Health Research Policy and Systems, London, v. 11, n. 1, p. 1-6, 2013. Artigo 3.

MACKENBACH, J. P.; MACKEE, M. Government, politics and health policy: a quantitative analysis of 30 European countries. Health Policy, Armsterdam, v. 119, n. 10, p. 1298-1308, 2015.

MALUKA, S. et al. Decentralized health care priority-setting in Tanzania: evaluating against the accountability for reasonableness framework. Social Science \& Medicine, New York, v. 71, n. 4, p. 751-759, 2010.

MALUKA, S. et al. Decentralization and health care prioritization process in Tanzania: from national rhetoric to local reality. The International Journal of Health Planning Management, Hoboken, v. 26, n. 2, p. 102-120, 2011 a.

MALUKA, S. et al. Implementing accountability for reasonableness framework at district level in Tanzania: a realist evaluation. Implementation Science, London, v. 6, p. 1-15, 2011b. Artigo 11.

MILAT, A. J. et al. Increasing the scale and adoption of population health interventions: experiences and perspectives of policy makers, practitioners, and researchers. Health Research Policy Policy and Systems, London, v. 12, p. 1-11, 2014. Artigo 18.

MUBYAZI, G. et al. Implications of decentralization for the control of tropical diseases in Tanzania: a case study of four districts. International Journal of Health Planning Management, Hoboken, v. 19, p. s167-185, 2004. Suplemento 1.

NUTLEY, T.; MACNABB, S.; SALETINE, S. Impact of a decision-support tool on decision making at the district level in Kenya. Health Research Policy and Systems, London, v. 12, p. 11-34, 2013. Artigo 18.

PHELPS, C. et al. Strategic Planning in Population Health and Public Health Practice: A Call to Action for Higher Education. Milbank Quarterly, New York, v. 94, n. 1, p. 109-125, 2016.

SANTOS, I. O mix público-privado no sistema de saúde brasileiro: elementos para a regulação da cobertura duplicada. 2009. 189 f. Tese (Doutorado em Saúde Pública) - Escola Nacional de Saúde Pública Sergio Arouca, Fundação Oswaldo Cruz, Rio de Janeiro, 2009.

SCHROEDER, S. M. Changing population health policy: a model for identifying health needs and recommendations to improve health status.

Population Health Management, v. 19, n. 3, p. 216 223, 2016.

TANIOS, N. et al. Which criteria are considered in healthcare decisions? Insights from an international survey of policy and clinical decision makers. International Journal of Technology Assessment in Health Care, Cambridge, v. 29, n. 4, p. 456-465, 2013.

WICKREMASINGHE, D. et al. District decisionmaking for health in low income settings: a systematic literature review. Health Policy and Planning, Oxford, v. 31, p. ii12-ii24, 2016. Suplemento 2.
Recebido: $11 / 07 / 2017$

Reapresentado: 24/11/2017

Aprovado: 06/06/2018 